\title{
Ischaemic stroke in young adults: predictors of outcome and recurrence
}

\author{
K Nedeltchev, T A der Maur, D Georgiadis, M Arnold, V Caso, H P Mattle, G Schroth, L Remonda, \\ M Sturzenegger, U Fischer, R W Baumgartner
}

J Neurol Neurosurg Psychiatry 2005;76:191-195. doi: 10.1136/jnnp.2004.040543

See end of article for authors' affiliations

.....................

Correspondence to: Dr Ralf W Baumgartner, Department of Neurology, University Hospital of Zürich, Fravenklinikstrasse 26, CH-8091 Zürich,

Switzerland; ralf.baumgartner@ nos.usz.ch

Received 1 March 2004 In revised form 19 May 2004

Accepted 24 May 2004

Background: There is limited information about predictors of outcome and recurrence of ischaemic stroke affecting young adults.

Objective: To assess the predictive value of the presenting characteristics for both outcome and recurrence in young stroke victims.

Methods: Clinical and radiological data for 203 patients aged 16 to 45 years were collected prospectively; they comprised $11 \%$ of 1809 consecutive patients with ischaemic stroke. The National Institutes of Health stroke scale (NIHSS), the Bamford criteria, and the trial of ORG 10172 in acute stroke treatment (TOAST) classification were used to define stroke severity, subtype, and aetiology. The clinical outcome of 198 patients (98\%) was assessed using the modified Rankin scale (mRS) and categorised as favourable (score 0-1) or unfavourable (score 2-6).

Results: Stroke was caused by atherosclerotic large artery disease in $4 \%$, cardioembolism in $24 \%$, small vessel disease in $9 \%$, another determined aetiology in 30\%, and undetermined aetiology in 33\%. Clinical outcome at three months was favourable in $68 \%$, unfavourable in $29 \%$, and lethal in $3 \%$. Thirteen non-fatal stroke, two fatal strokes, and six transient ischaemic attacks (TIA) occurred during a mean (SD) follow up of 26 (17) months. High NIHSS score, total anterior circulation stroke, and diabetes mellitus were independent predictors of unfavourable outcome or death $(p<0.0001, p=0.011$, and $p=0.023)$. History of TIA predicted stroke recurrence $(p=0.02)$.

Conclusions: Severe neurological deficits at presentation, total anterior circulation stroke, and diabetes mellitus predict unfavourable outcome. Previous TIA are associated with increased risk of recurrence.

$\mathrm{S}$ troke in young adults is rare but can be devastating for the affected individuals and their families. Recently, there has been increasing interest in this topic owing to increasing stroke rates in the younger age group and improved patient evaluation and treatment options. ${ }^{1-6}$

There is general agreement that young adults have a better chances of surviving a stroke than older individuals. However, the majority of survivors have emotional, social, or physical sequelae that impair their quality of life. ${ }^{8}$ In addition, young stroke victims are responsible for providing child care or generating income for their families. Therefore, the ability to predict prognosis would be of paramount importance in this patient population.

Few studies have examined the ability of clinical, laboratory, or imaging variables to predict the clinical outcome of ischaemic stroke and the risk of stroke recurrence in young adults. $^{8-11}$ The severity of the initial neurological deficit was the only reproducible predictor of outcome in these studies. Age over 35 years, male sex, and cardiac diseases were independent predictors of recurrent stroke, myocardial infarction, or death from any cause in a single study, ${ }^{11}$ but had no predictive value for the outcome in another. ${ }^{10}$

Our aim in this study was to evaluate the ability of presenting clinical, laboratory, and radiological characteristics to predict the clinical outcome and recurrence of ischaemic stroke in young adults.

\section{METHODS}

This study is based on the data from the Zurich and Bern ischaemic stroke registries, which were set up in August 1997 and January 2000, respectively. Both registries use systematic computer coding of prospectively collected data from consecutive patients admitted with ischaemic stroke to the stroke units of two university hospitals, which have a catchment area of about 2140000 inhabitants. This study comprises all patients with acute ischaemic stroke who were younger than 45 years of age, admitted in Zurich from August 1997 to October 2002, and in Bern from January 2000 to September 2002.

The diagnosis of ischaemic stroke was based on a focal neurological deficit that lasted for at least 24 hours with or without corresponding ischaemic lesions on brain imaging. ${ }^{12}$ The TOAST classification (trial of ORG 10172 in acute stroke treatment) ${ }^{13}$ was used to define the aetiology of stroke:

- large artery atherosclerosis;

- cardioembolism;

- small vessel disease;

- stroke of other determined aetiology;

- stroke of undetermined aetiology.

Furthermore, strokes were classified according to the Bamford criteria in total anterior circulation stroke (TACS), partial anterior circulation stroke (PACS), posterior circulation stroke (POCS), and lacunar stroke (LACS). ${ }^{14}$

The following stroke risk factors were assessed: sex, hypertension (defined by preadmission history and medical records), diabetes mellitus (defined by venous plasma glucose concentration of $\geqslant 7.0 \mathrm{mmol} / \mathrm{l}$ after an overnight fast on

Abbreviations: LACS, lacunar stroke; mRS, modified Rankin scale; NIHSS, National Institutes of Health stroke scale; PACS, partial anterior circulation stroke; POCS, posterior circulation stroke; TACS, total anterior circulation stroke; TOAST, trial of ORG 10172 in acute stroke treatment 
at least two separate occasions, and/or $\geqslant 11.1 \mathrm{mmol} / \mathrm{l}$ two hours after the ingestion of $75 \mathrm{~g}$ of oral glucose and on one other occasion during the two hour test), current cigarette smoking, hypercholesterolaemia (defined as a total venous plasma cholesterol concentration greater than $5 \mathrm{mmol} / \mathrm{l}$ ), heart disease, history of amaurosis fugax (monocular blindness lasting $<24$ hours), retinal infarct (monocular blindness lasting $\geqslant 24$ hours), transient ischaemic attack (TIA, neurological deficit lasting $<24$ hours) or ischaemic stroke, migraine (diagnosed in accordance with the criteria of the International Headache Society ${ }^{15}$ ), and use of oral contraceptives.

The severity of the neurological deficit on admission was assessed using the National Institutes of Health stroke scale (NIHSS) ${ }^{16}$

All patients were investigated using a standard protocol including blood tests (determination of red and white blood cell and platelet counts, glucose, cholesterol, electrolytes, transaminases, creatinine, urea, prothrombin time, activated partial thromboplastin time), cranial computed tomography (CT) and/or conventional magnetic resonance imaging (MRI) ( 1.5 Tesla system providing axial T1, T2, and proton density weighted images, and gadolinium-diethylentriamine-pentaacetic acid enhanced T1 weighted images), 12 lead electrocardiography, and ultrasound evaluation of the brain supplying arteries. The following additional investigations were carried out at the discretion of the treating physician: a search for vasculitis including antiphospholipid antibodies, C reactive protein, transthoracic and transoesophageal echocardiography, 24 hour Holter monitoring, cervical MRI with fat suppression, diffusion weighted and perfusion weighted MRI, and magnetic resonance or catheter angiography.

The three month clinical outcome was evaluated using the modified Rankin scale (mRS) score. ${ }^{17}$ The mRS score was used to classify clinical outcome as favourable (score 0-1) or unfavourable (score 2-6). Information on recurrent cerebral and retinal ischaemic events was gathered thoroughly during annual follow up visits or structured telephone interviews. The follow up included a clinical visit in 146 cases and a structured telephone interview in 52.

\section{Statistics}

Statistical analysis was carried out using SPSS 10 for MacIntosh statistical software ${ }^{\odot}, 2001$ (SPSS Inc). The Mann-Whitney U test was done to examine the association between baseline factors and clinical outcome individually. In order to identify variables that might indicate a poor prognosis, outcome was dichotomised into favourable (mRS score 0-1) and unfavourable or death (mRS score 2-6). A logistic regression analysis was undertaken including the variables that showed significant relations with outcome

Table 1 Presenting characteristics in 203 young adults with ischaemic stroke (mean (SD) age 36 (8) years)

\begin{tabular}{lc}
\hline Characteristic & $\mathbf{n}(\%)$ \\
\hline Men & $108(53)$ \\
Hypertension & $38(19)$ \\
Diabetes mellitus & $4(2)$ \\
Smoking & $94(46)$ \\
Hypercholesterolaemia & $79(39)$ \\
Coronary artery disease & $3(1)$ \\
History of transient ischaemic attacks & $24(12)$ \\
History of ischaemic stroke & $8(4)$ \\
History of amaurosis fugax & $6(3)$ \\
History of migraine without aura & $37(18)$ \\
C reactive protein level $>5$ mg/l & $73(36)$ \\
Oral contraceptives & $21\left(22^{*}\right)$ \\
\hline &
\end{tabular}

when tested individually. Probability (p) values of less than 0.05 were considered statistically significant. Kaplan-Meier survival data analysis was used to investigate whether or not the risk of recurrent cerebral ischaemic events varied with time and previous TIA. Recurrent stroke (both fatal and nonfatal) and TIA were considered end points of follow up, whereas death from any other cause was a censoring event. Average annual event rates were calculated according to the formula $1-(1-P)^{1 / n}$, where $P$ is the cumulative event rate at $n$ years of follow up. The log-rank test was used for univariate analysis of independent variables (age, sex, stroke risk factors, aetiology, and subtype) with regard to their association with the rate of recurrence.

\section{RESULTS}

The study included 136 young adults from among 1105 patients with ischaemic stroke in Zurich (12\%; 80 men, 56 women), and 67 of 704 in Bern (10\%; 28 men, 39 women). Thus the study population consisted of 203 patients (108 men, 95 women) aged between 16 and 45 years (mean (SD), 36 (8) years; table 1).

One hundred and sixty two patients $(80 \%)$ had at least one stroke risk factor, $106(52 \%)$ had two or more. Cigarette smoking, hypercholesterolaemia, and hypertension were detected in 19\%, 39\%, and $46 \%$ of cases, while diabetes mellitus and coronary artery disease were rare $(2 \%$ and $1 \%$, respectively). Thirty eight patients (19\%) had a well documented history of retinal or cerebral ischaemia. Migraine showed a non-significant trend to be more prevalent among women $(22 \%, \mathrm{n}=21)$ than men $(15 \%, \mathrm{n}=16)(\mathrm{p}=0.056)$.

Stroke aetiology is shown in table 2. Most strokes were caused by cardiac embolism and cervical artery dissection, whereas only $9 \%$ and $4 \%$ resulted from small vessel disease and large artery atherosclerosis, respectively. The most frequent abnormality found in patients with cardioembolic stroke was a patent foramen ovale. This became evident in 43 of the 48 patients and was associated with an atrial septal aneurysm in 11 patients. Atrial septal defect was diagnosed in three patients, intermittent atrial fibrillation, left atrial thrombus in mitral stenosis, anterior myocardial infarction, subacute endocarditis, cardiomyopathy related to Duchenne muscular dystrophy, and endomyocardial fibrosis in one patient each. The cause of stroke remained undetermined in a third of the cases.

The median NIHSS score on admission was 5 (range 1 to 35) (table 3). Patients with total anterior circulation strokes had more severe neurological deficits than those with posterior circulation $(p=0.0002)$ or lacunar strokes $(p<0.0001)$.

\begin{tabular}{lc}
$\begin{array}{l}\text { Table } 2 \text { Stroke aetiology in } 203 \text { young adults with } \\
\text { ischaemic stroke }\end{array}$ \\
\hline Aetiology & $\mathrm{n}(\%)$ \\
\hline Large artery atherosclerosis & $8(4)$ \\
Cardiac embolism & $48(24)$ \\
Small vessel disease & $18(9)$ \\
Other determined aetiology & $61(30)$ \\
Cervical artery dissection & $48(24)$ \\
Migrainous infarction & $2(0.9)$ \\
Essential thrombocythemia & $2(0.9)$ \\
Factor V Leiden deficiency & $2(0.9)$ \\
Complication of catheter coronary angiography & $2(0.9)$ \\
Systemic lupus erythematosus & $1(0.5)$ \\
Cocaine use & $1(0.5)$ \\
Protein C deficiency & $1(0.5)$ \\
Fabry's disease & $1(0.5)$ \\
Eclampsia & $1(0.5)$ \\
Undetermined aetiology & $68(33)$ \\
\hline
\end{tabular}


Partial anterior circulation strokes caused more severe neurological symptoms than lacunar strokes $(p=0.003)$.

Information on clinical outcome at three months was available for 198 of 203 patients (98\%): 63 of 67 from Bern (94\%) and 135 of 136 from Zurich (99\%). Five patients were lost to follow up because they had moved without indicating their new address. The clinical outcome was favourable in 135 of 198 patients $(68 \%)$, and unfavourable in the remaining 63 cases $(32 \%)$, in whom the mRS was $2-5$ in 58 patients $(29 \%)$ and 6 in five patients $(3 \%)$.

Most patients with LACS ( $88 \%$ ), but only $28 \%$ with TACS, had a favourable clinical outcome (table 3). Patients with PACS or POCS had a favourable clinical outcome in $74 \%$ and $69 \%$, respectively. Five of 203 patients (2\%) with TACS $(n=2)$ or POCS $(n=3)$ died from their index stroke, all within seven days from stroke onset. Lethal stroke was caused by cardiac embolism and vertebral artery dissection in one patient each, and the cause remained undetermined in the other three patients.

Logistic regression analysis showed that a history of diabetes mellitus, a high NIHSS score on admission, and TACS were independent predictors of an unfavourable outcome $(p<0.0001, p=0.011$, and $p=0.023$, respectively $)$. Age, sex, stroke risk factors other than diabetes mellitus, and stroke aetiology had no predictive value for the clinical outcome.

Fifteen strokes and six TIA occurred during a mean (SD) follow up of 26 (17) months (439 patient-years). Among the 15 patients with recurrent strokes, the aetiology of the index event was large artery atherosclerosis in two patients, cardiac embolism in four, carotid artery dissection in two, and unknown in seven. Recurrent TIA occurred in patients with large artery atherosclerosis (1), essential thrombocythemia (1), cardiac embolism (1), and stroke of undetermined aetiology (2). Two patients who had suffered a TACS of undetermined aetiology died from their recurrent stroke at, respectively, 12 and 66 months after the index event. Six patients who had recovered from the index stroke worsened to an unfavourable outcome. The log-rank test for survival data did not show an association between age, sex, stroke risk factors other than previous TIA, stroke aetiology, stroke subtype, or the risk for recurrence.

At two years of follow up, the overall cumulative estimate of risk of stroke in the whole cohort was $6.0 \% \quad(95 \%$ confidence interval, $4.1 \%$ to $7.9 \%$ ), and that of stroke or TIA, $11.4 \%(8.7 \%$ to $14.0 \%)$. The annual rate for stroke recurrence was $3.0 \%$, and the combined rate for stroke and TIA was $5.9 \%$. When patients with a history of TIA were analysed separately, the average annual rate of recurrent stroke was $11.7 \%$, and that of stroke and TIA was $15.5 \%$. The log-rank test for survival data showed a significantly higher risk for recurrent events in this group than in patients without TIA before the qualifying stroke $(p=0.02)$ (fig 1 ).

\section{DISCUSSION}

Our study evaluated predictors of clinical outcome and recurrence of ischaemic stroke in 203 young adults. Clinical outcome was favourable in $68 \%$ and unfavourable in the remaining cases, including $3 \%$ of deaths. In a multivariate regression model, a history of diabetes mellitus, initial stroke severity, and total anterior circulation strokes were associated with an unfavourable clinical outcome. The annual risk of stroke recurrence was $3.0 \%$ for all patients and $11.7 \%$ for patients with a history of TIA, which was associated with increased risk of stroke recurrence.

The patients enrolled in this study constituted $10 \%$ and $12 \%$ of all patients with acute ischaemic stroke who were admitted to the stroke units of the two university centres. This rate is higher than that reported in population based studies. In industrialised nations, fewer than $5 \%$ of all strokes occur in subjects younger than 45 years of age. ${ }^{14}$ Thus the higher rate of young adults with stroke found in this study probably reflects referral bias, because younger patients are more likely to be referred to a centre with a stroke unit. This assumption is in accordance with the results of referral based studies, in which the proportion of young compared with older stroke patients was similar to our findings. ${ }^{18}$

The sex distribution of the Zurich patients differed from that of the Bernese patients (men $v$ women, $80 v 56$ in Zurich and $28 v 39$ in Bern). The proportion of young adults $(<45$ years of age) among all patients admitted with ischaemic stroke was similar in the two centres, which indicates that referral bias is not a likely explanation for the difference in sex distribution. Zurich and Bern are university based tertiary care centres with a similar stroke expertise, catchment area, and admissions policy, and the number of patients included per centre was relatively low. These findings suggest that the difference in sex distribution observed in this study was likely to have been due to chance. The prevalence of stroke risk factors and their distribution among the two sexes was similar to the findings reported in other studies evaluating young adults. ${ }^{319}$ Current smoking was the most prevalent risk factor, followed by hypercholesterolaemia, family history of cerebrovascular disease, and hypertension. Two or more risk factors were found in $52 \%$ of our patients, which is similar to the $61 \%$ reported in young adult Italians with ischaemic stroke. ${ }^{19}$

The aetiology of stroke differs in young compared with older patients. ${ }^{20}$ Correspondingly, cardiac embolism and other determined aetiologies-especially cervical artery dissection-were found in 54\%, and atherothrombosis and small vessel disease in $13 \%$ of our patients. Furthermore, our

Table 3 Initial stroke severity and clinical outcome in 203 young adults with ischaemic stroke

\begin{tabular}{|c|c|c|c|c|}
\hline & \multirow[b]{2}{*}{$\begin{array}{l}\text { Median } \\
\text { NIHSS }\end{array}$} & \multicolumn{3}{|c|}{ Clinical outcome (n (\%)) } \\
\hline & & $\begin{array}{l}\text { Favourable } \\
\text { (mRS score 0-1) }\end{array}$ & $\begin{array}{l}\text { Unfavourable } \\
\text { (mRS score 2-5) }\end{array}$ & Death (mRS score 6) \\
\hline TACS $(n=31)$ & 13 & $8(28)$ & $19(65)$ & $2(7)$ \\
\hline PACS $(n=80)$ & 5 & $57(74)$ & $20(26)$ & 0 \\
\hline LACS $(\mathrm{n}=34)$ & 3 & $30(88)$ & $4(12)$ & 0 \\
\hline POCS $(\mathrm{n}=58)$ & 3.5 & $40(69)$ & $15(26)$ & $3(5)$ \\
\hline Total $(n=203)^{*}$ & 5 & $135(68)$ & $58(29)$ & $5(3)$ \\
\hline
\end{tabular}

*Outcome at three months was assessed in 198 of the 203 patients, because five were lost to follow up (two TACS and three PACS).

LACS, lacunar stroke; mRS, modified Rankin scale; NIHSS, National Institutes of Health stroke scale; PACS, partial anterior circulation stroke; POCS; posterior circulation stroke; TACS, total anterior circulation stroke. 


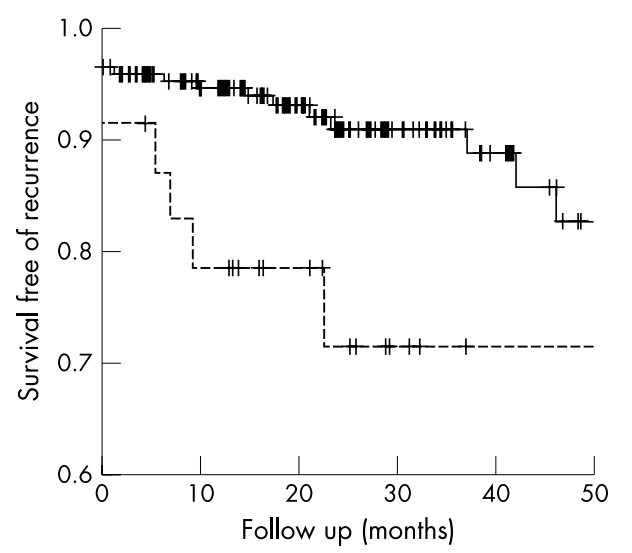

Figure 1 Kaplan-Meier survival curves of patients without a history of transient ischaemic attack (TIA) and those with TIA before the qualifying stroke. The $x$ axis indicates the time since the index ischaemic event. The $y$ axis indicates the proportion of patients surviving free of recurrence. The dotted line represents the patients with history of TIA before the qualifying stroke.

study confirms the greater heterogeneity in stroke aetiology found in strokes affecting young adults (table 2). ${ }^{121}$ Every third patient suffered from a stroke of other determined aetiology according to the TOAST classification. ${ }^{13}$ We identified 10 different aetiologies within this stroke category, cervical artery dissection being the most frequent. The aetiological diversity of stroke in young adults is often challenging. On the one hand, numerous investigations and tests have to be carried out in order to consider all possible aetiologies; on the other hand, needless examinations should be avoided by planning the diagnostic evaluation on a case by case basis. ${ }^{72}$

The main limitation of this study was the relatively small number of patients examined. This reflected the low prevalence of ischaemic stroke in young adults. It must be taken into account that the patients enrolled represented the total number of cases presenting over a period of nearly eight years in two university hospitals with a catchment area of more than two million inhabitants.

The clinical outcome in patients with ischaemic stroke is usually classified as favourable and unfavourable when the mRS scores are 0-2 and 3-6, respectively. ${ }^{23}{ }^{24}$ While the ability to look after one's own affairs without assistance (mRS score of 2) would mean a favourable outcome for elderly people, this might not be the case for young adults. Thus we defined favourable outcome as no symptoms at all (mRS score of 0) or no significant disability despite symptoms (mRS score of 1). In our series, a favourable outcome was found in $68 \%$ of the patients. The published data on clinical outcome in ischaemic stroke in young adults are discordant. Leys et al reported that $87 \%$ of young adults with ischaemic stroke became independent, defined by an mRS of $0-2 .{ }^{25}$ Seventy eight per cent of all patients and $84 \%$ of the survivors had an mRS score of 0 or 1 . Conversely, Neau et $a l^{8}$ observed that the quality of life remained moderately deteriorated or poor in $43 \%$ of stroke patients under 45 years of age. The outcome of the cases reported by Rozenthul-Sorokin et al was even worse, as only $10.8 \%$ had a favourable outcome (mRS 0-1) and $16 \%$ became independent (mRS 0-2). ${ }^{2}$

To date, only a few studies have assessed the value of presenting characteristics to predict clinical outcome and recurrence of ischaemic stroke in young adults. ${ }^{8-11}$ The severity of the neurological deficit found on admission was the major predictor of clinical outcome in three studies. ${ }^{8-10}$ In addition, absence of dysphasia, left hemiplegia, and age less than 40 years were associated with a better functional outcome in the study by Kong et al. ${ }^{10}$ Our results confirmed the relevance of the severity of the presenting neurological deficit assessed by the NIHSS as an independent predictor of unfavourable outcome, as defined by an mRS score of 2-5. Furthermore, the combination of weakness (eventually accompanied by sensory deficit) of at least two of three body areas, homonymous hemianopia, and higher cerebral dysfunction was independently associated with an unfavourable outcome. Diabetes mellitus, even though present in only $2 \%$ of the patients in this cohort, was an independent predictor of unfavourable outcome in a multivariate regression model. This finding is in accordance with the findings observed in adult stroke patients with diabetes. ${ }^{26}$

The annual risk of stroke recurrence was $3.0 \%$ and the combined risk for stroke and TIA was $5.9 \%$. This risk is considerably lower than in older stroke patients. ${ }^{27-29}$ However, the stroke risk found in this series was substantially higher than the rates observed in young stroke patients by Leys et al, which was $1.4 \%$ during the first year and $1.0 \%$ in the subsequent years. ${ }^{25}$

A history of TIA was associated with an increased risk of stroke recurrence in this series. Transient ischaemic attacks have been recognised as a potent risk factor for future strokes in older patients. ${ }^{30}$ After a first TIA, $10-20 \%$ of adult patients will suffer a stroke within the next 90 days. ${ }^{31}$ Our study is the first to show that the increased risk of stroke recurrence applies also to patients under 45 years of age.

\section{Conclusions}

Our data suggest that stroke in young adults is not as benign as previously suggested, as a favourable outcome was observed in two thirds of cases and the annual stroke rate was $3 \%$. Predictors of an unfavourable outcome were a history of diabetes mellitus, a severe neurological deficit at presentation, and total anterior circulation stroke, while a history of transient ischaemic attacks was associated with an increased risk of stroke recurrence.

\section{ACKNOWLEDGEMENTS}

We thank Pietro Ballinari PhD for statistical advice. This study is funded in part by a grant from the Swiss National Science Foundation (SNF 3100-66348.01)

\section{Authors' affiliations}

K Nedeltchev, T A der Maur, M Arnold, H P Mattle, M Sturzenegger, U Fischer, Department of Neurology, University Hospital of Bern, Switzerland

D Georgiadis, V Caso, R W Baumgartner, Department of Neurology, University Hospital of Zürich, Switzerland

G Schroth, L Remonda, Department of Neuroradiology, University Hospital of Bern, Switzerland

Competing interests: none declared

\section{REFERENCES}

1 Nencini $\mathbf{P}$, Inzitari $D$, Baruffi MC, et al. Incidence of stroke in young adults in Florence, Italy. Stroke 1988;19:977-81.

2 Rozenthul-Sorokin N, Ronen R, Tamir A, et al. Stroke in the young in Israel. Incidence and outcomes. Stroke 1996;27:838-41.

3 Kristensen B, Malm J, Carlberg B, et al. Epidemiology and etiology of ischemic stroke in young adults aged 18 to 44 years in northern Sweden. Stroke 1997;28:1702-9

4 Marini C, Totaro R, De Santis F, et al. Stroke in young adults in the communitybased L'Aquila registry: incidence and prognosis. Stroke 2001;32:52-6.

5 Jacobs BS, Boden-Albala B, Lin IF, et al. Stroke in the young in the northern Manhattan stroke study. Stroke 2002;33:2789-93.

6 Naess H, Nyland HI, Thomassen L, et al. Incidence and short-term outcome of cerebral infarction in young adults in western Norway. Stroke 2002;33:2105-8

7 Kappelle $\amalg$, Adams HPJ, Heffner ML, et al. Prognosis of young adults with ischemic stroke. A long-term follow-up study assessing recurrent vascular events and functional outcome in the lowa registry of stroke in young adults. Stroke 1994;25:1360-5 
8 Neau JP, Ingrand P, Mouille-Brachet C, et al. Functional recovery and social outcome after cerebral infarction in young adults. Cerebrovasc Dis 1998;8:296-302.

9 Ferro JM, Crespo M. Prognosis after transient ischemic attack and ischemic stroke in young adults. Stroke 1994;25:1611-16.

10 Kong KH, Chan KF, Tan ES. Functional outcome in young strokes. Ann Acad Med Singapore 1995;24:172-6.

11 Marini C, Totaro R, Carolei A. Long-term prognosis of cerebral ischemia in young adults. National research council study group on stroke in the young. Stroke 1999:30:2320-5.

12 Aho K, Harmsen P, Hatano S, et al. Cerebrovascular disease in the community: results of a WHO collaborative study. Bull WHO 1980;58:113-30.

13 Adams HPJ, Bendixen BH, Kappelle $\sqcup$, et al. Classification of subtype of acute ischemic stroke. Definitions for use in a multicenter clinical trial. TOAST. Trial of Org 10172 in acute stroke treatment. Stroke 1993;24:35-41.

14 Bamford J, Sandercock P, Dennis M, et al. Classification and natural history of clinically identifiable subtypes of cerebral infarction. Lancet 1991;337:1521-6.

15 Classification and diagnostic criteria for headache disorders, cranial neuralgias and facial pain. Headache classification committee of the international headache society. Cephalalgia 1988;8(suppl 7):1-96.

16 Brott T, Marler JR, Olinger CP, et al. Measurements of acute cerebral infarction: lesion size by computed tomography. Stroke 1989;20:871-5.

17 van Swieten JC, Koudstaal PJ, Visser MC, et al. Interobserver agreement for the assessment of handicap in stroke patients. Stroke 1988;19:604-7.

18 Bogousslavsky J, Van Melle G, Regli F. The Lausanne stroke registry: Analysis of 1000 consecutive patients with first stroke. Stroke 1988;19:1083-92.

19 Musolino R, La Spina P, Granata A, et al. Ischaemic stroke in young people: a prospective and long-term follow-up study. Cerebrovasc Dis 2003;15:121-8.

20 Zeiler K, Siostrzonek P, Lang W, et al. Different risk factor profiles in young and elderly stroke patients with special reference to cardiac disorders. J Clin Epidemiol 1992; 45:1383-9.
21 Blecic S, Bogousslavsky J. Stroke in young adults. In: Barnett HJ, et al, eds. Stroke: pathophysiology, diagnosis and management. New York: Churchill Livingstone, 1998:1001-12.

22 Adams HP, Kappelle $\sqcup$, Biller J, et al. Ischemic stroke in young adults. Experience in 329 patients enrolled in the lowa registry of stroke in young adults. Arch Neurol 1995;52:491-5.

23 Furlan A, Higashida R, Wechsler L, et al, for the PROACT investigators. Intraarterial prourokinase for acute ischemic stroke: the PROACT II study: a randomized controlled trial: prolyse in acute cerebral thromboembolism. JAMA 1999;282:2003-11.

24 Arnold M, Schroth G, Nedeltchev K, et al. Intra-arterial thrombolysis in 100 patients with acute stroke due to middle cerebral artery occlusion. Stroke 2002;33:1828-33.

25 Leys D, Bandu L, Henon $\mathrm{H}$, et al. Clinical outcome in 287 consecutive young adults ( 15 to 45 years) with ischemic stroke. Neurology 2002;59:26-33.

26 Weimar C, Ziegler A, Konig IR, et al. Predicting functional outcome and survival after acute ischemic stroke. J Neurol 2002;249:888-95.

27 Secondary prevention in non-rheumatic atrial fibrillation after transient ischaemic attack or minor stroke. EAFT (European Atrial Fibrillation Trial) study group. Lancet 1993;342:1255-62.

28 A randomised, blinded, trial of clopidogrel versus aspirin in patients at risk of ischaemic events (CAPRIE). CAPRIE steering committee. Lancet 1996:348:1329-39.

29 Mohr JP, Thompson JL, Lazar RM, et al, for the Warfarin-Aspirin Recurrent Stroke Study Group. A comparison of warfarin and aspirin for the prevention of recurrent ischemic stroke. N Engl J Med 2001;345:1444-51.

30 Albers GW, Caplan LR, Easton JD, et al. Transient ischemic attack - proposal for a new definition. N Engl J Med 2002;347:1713-16.

31 Johnston SC, Gress DR, Browner WS, et al. Short-term prognosis after emergency department diagnosis of TIA. JAMA 2000;284:2901-6.

Committee on Publication Ethics Seminar 2005

\section{Friday 11 March 2005, 9.30 am - 5 pm, BMA House, London}

This year's seminar will focus on COPE's new Code of Conduct for Editors and interactive workshops on common ethical and editorial dilemmas. The seminar is for editors, authors, and all those interested in increasing the standard of publication ethics.

The Code aims to set a new basic standard for the ethical conduct of editors and sets out guidelines for quality and correcting the record, standing by decisions made, ethics committee approval, consent for publication confidentiality of submitted material, guidance to authors, pursuing misconduct, relationship to publishers, owners, and advertisers, and conflict of interest. The code also creates a mechanism to refer a complaint to COPE if an editor has breached the code.

The seminar will include:

- The new Code of Conduct for Editors

- Dr lona Heath, Chair BMJ Ethics Committee-research, audit, and ethics committee approval

- COPE's new website-full text and keyword searching for COPE's advice on specific issues, for example research misconduct, conflict of interest, and deception

- Interactive workshops-common ethical and editorial dilemmas for editors

- Opportunities to network with other editors and share your experiences and challenges

The seminar is free for COPE members and $£ 30.00$ for non-members. Numbers are limited and early booking is advisable. For registrations or more information please contact Sam Knottenbelt at cope@bmigroup.com or call 0207383 6602. For more information on COPE see www. publicationethics.org.uk/ 